\title{
Immunomodulatory Approaches in Diabetes-Induced Cardiorenal Syndromes
}

\author{
Lama A. Ammar ${ }^{1,2 \dagger}$, Mohamad I. Nahlawi ${ }^{1,2 \dagger}$, Nizar W. Shayya ${ }^{1,2 \dagger}$, Hilda E. Ghadieh ${ }^{1,2+}$, \\ Nadim S. Azar ${ }^{1,2}$, Frédéric Harb ${ }^{3 *}$ and Assaad A. Eid ${ }^{1,2 *}$ \\ ${ }^{1}$ Department of Anatomy, Cell Biology and Physiological Sciences, Faculty of Medicine and Medical Center, American \\ University of Beirut, Beirut, Lebanon, ${ }^{2}$ American University of Beirut Diabetes, American University of Beirut, Beirut, Lebanon, \\ ${ }^{3}$ Department of Life and Earth Sciences, Faculty of Sciences, Lebanese University, Fanar, Lebanon
}

Immunomodulatory approaches are defined as all interventions that modulate and curb the immune response of the host rather than targeting the disease itself with the aim of disease prevention or treatment. A better understanding of the immune system continues

OPEN ACCESS

Edited by:

George W. Booz

University of Mississippi Medical Center School of Dentistry,

United States

Reviewed by:

Bassam Badran,

Lebanese University, Lebanon Nassim Fares,

Saint Joseph University, Lebanon

*Correspondence:

Assaad A. Eid

ae49@aub.edu.lb

Frédéric Harb

frederic.harb@ul.edu.lb

tThese authors have contributed equally to this work

Specialty section:

This article was submitted to Cardiovascular Therapeutics,

a section of the journal

Frontiers in Cardiovascular Medicine

Received: 18 November 2020

Accepted: 31 December 2020

Published: 28 January 2021

Citation:

Ammar LA, Nahlawi MI, Shayya NW,

Ghadieh HE, Azar NS, Harb F and Eid AA (2021) Immunomodulatory Approaches in Diabetes-Induced

Cardiorenal Syndromes. Front. Cardiovasc. Med. 7:630917. doi: 10.3389/fcvm.2020.630917 to offer innovative drug targets and methods for immunomodulatory interventions. Cardiorenal syndrome is a clinical condition that defines disorders of the heart and kidneys, both of which communicate with one another through multiple pathways in an interdependent relationship. Cardiorenal syndrome denotes the confluence of heart-kidney relationships across numerous interfaces. As such, a dysfunctional heart or kidney has the capacity to initiate disease in the other organ via common hemodynamic, neurohormonal, immunological, and/or biochemical feedback pathways. Understanding how immunomodulatory approaches are implemented in diabetesinduced cardiovascular and renal diseases is important for a promising regenerative medicine, which is the process of replacing cells, tissues or organs to establish normal function. In this article, after a brief introduction on the immunomodulatory approaches in diseases, we will be reviewing the epidemiology and classifications of cardiorenal syndrome. We will be emphasizing on the hemodynamic factors and nonhemodynamic factors linking the heart and the kidneys. In addition, we will be elaborating on the immunomodulatory pathways involved in diabetes-induced cardiorenal syndrome namely, RAS, JAK/STAT, and oxidative stress. Moreover, we will be addressing possible therapeutic approaches that target the former pathways in an attempt to modulate the immune system.

Keywords: cardiorenal syndromes, diabetes mellitus, ras pathway, JAK/STAT pathway, oxidative stress, immunomodulatory approaches

\section{IMMUNOMODULATORY APPROACHES IN DISEASES}

Immunomodulatory approaches are defined as the all interventions that modulate and curb the immune response of the host rather than targeting the disease itself (1). With the ongoing attempts of treating infectious diseases amidst the increased pathogen resistance to traditional infectious disease control approaches, immunomodulatory interventions are being closely reviewed (1). Immunomodulatory medicines alter the response of the immune system by increasing or decreasing the production of serum antibodies, using immunostimulators and 
immunosuppressives, respectively (2). Immunostimulators are administered in order to enhance the immune response against infectious diseases, tumors, primary or secondary immunodeficiency, and alterations in antibody transfer. However, immunosuppressive drugs are used to reduce the immune response against transplanted organs and to treat autoimmune diseases (2). The ability of immunomodulatory approaches to modulate the immune system's disease response is evidently credited to the disruption of the proinflammatory cascade through various mechanisms involving the antioxidants effects, disruption of bacterial flora, monoclonal antibodies, cytokines, and related extracellular immune mediators and alterations in cell signaling (3). Hence, selectively either inhibiting or intensifying the specific populations and subpopulations of immune responsive cells (2).

Immunomodulatory approaches are heavily used in the medical field. In oncology, particularly for cancers unresponsive to known agents, antitumor immunotherapy is evidently decreasing fatalities through immune-cell-targeted monoclonal antibody (mAb) therapy and adoptive cellular therapy (ACT) (4). Immunomodulatory approaches have also gained attention in cardiovascular diseases by inducing inhibition to early inflammation initiators including reactive oxygen species, inhibition of mast cell degranulation and leukocyte infiltration and blocking the inflammatory cytokines and inhibiting the adaptive B and T-lymphocytes (5). That in addition to nephrology, where manipulation of the patients innate immune system, leads to the enhancement of renal repair and recovery of renal tissue hence, diminishing acute kidney injury without the progression to chronic renal diseases and consequently renal failure (6).

In this regard, understanding how immunomodulatory approaches are implemented in diabetes-induced cardiovascular and renal diseases is important for a promising regenerative medicine. Heart failure, particularly with preserved ejection

Abbreviations: CRS, Cardiorenal Syndrome; RAS, Renin Angiotensin System; JAK, Janus kinase; STAT, Signal Transducer And Activator of Transcription; DM, Diabetes Mellitus; MSC, Mesenchymal stem cells; DCM, Diabetic Cardiomyopathy; DN, Diabetic Nephropathy; mAb, Monoclonal antibody; ACT, Adoptive cellular therapy; HFpEF, Heart failure with preserved ejection fraction; FFAs, Free fatty acids; LGI, Low-grade inflammation; TNF- $\alpha$, Tumor necrosis factor-alpha; NF- $\kappa B$, Nuclear Factor Kappa B; TGF- $\beta$, Transforming Growth Factor-Beta; IFN- $\gamma$, Interferon-gamma; IL, Interleukin; Treg, T-regulatory cells; Th17, T-helper 17; CAD, Coronary artery disease; GFR, Glomerular filtration rate; GBM, Glomerular basement membrane; LXA4, Lipoxin 4; ADQI, Acute Dialysis Quality Initiative; HF, Heart failure; RBF, Renal blood flow; SNS, Sympathetic nervous system; NADPH, Nicotinamide Adenine Dinucleotide Phosphate; NOX, Nicotinamide Adenine Dinucleotide Phosphate oxidase; NOS, Nitric Oxide Synthase; ACE, Angiotensin Converting Enzyme; AT-1, Angiotensin II type 1 receptor; AT-2, Angiotensin II type 2 receptor; PKC, Protein Kinase C; AP1, Activator Protein 1; Cx43, Connexin 43; VCAM-1, Vascular Cell Adhesion Molecules-1; ICAM-1, Intracellular Adhesion Molecule-1; ET-1, Endothelin 1; TLR-47, Toll-Like Receptor 4; ERK, Extracellular Receptor Kinase; MAPK, Mitogen Activated Protein Kinase; COX-2, Cyclooxygenase 2; ROS, Reactive Oxygen Species; SOCS3, Cytokine Signaling 3; LPS, Lipopolysaccharide; CD14, Cluster of Differentiation 14; ADMA, Asymmetric Dimethyl Arginine; TxA2, Thromboxane A2; MMP, Matrix Metalloproteinase; XO, Xanthine Oxidase; EMT, Epithelial-Mesenchymal Transition; MAPK, Mitogen-Activated Protein kinase; CoQ10, Coenzyme Q10; STZ, Streptozotocin-induced; DCs, Dendritic cells; PD-1, Programmed death 1; QLQX, Qiliqiangxin. fraction (HFpEF) is highly prevalent in diabetic patients (7). Several factors increase the risk of heart failure in diabetic patients. These include abnormal cardiac handling of glucose and free fatty acids (FFAs), the effect of the metabolic derangements of diabetes on the cardiovascular system and the effect of most anti-diabetic agents with glucose-lowering molecules that have direct downregulations on the cardiovascular system (8). Consequently, careful assessment should be done on how to treat patients suffering from cardiac and renal disease concurrently. This is where immunomodulatory approaches take effect given that Diabetes Mellitus (DM) is an autoimmune disease with progressive status of chronic, low-grade inflammation (LGI) (9).

Mesenchymal stem cells (MSC) are gaining large interest as being a potential approach for both heart diseases and diabetes (10). MSC are multipotent stromal cells, nonhematopoietic progenitor cells that have shown to have wide immunomodulatory capabilities by altering the adaptive and innate immunity. These capabilities are due to the natural differentiating capacity of MSCs into various different cell lineages (11). When an autoimmune disease is the target, MSC modulate the immune system of the host by inhibiting the proliferation of $\mathrm{T}$ cells stimulated with either polyclonal mitogens (12), allogeneic cells or specific antigens (13) through inducing an arrest to the lymphocytes at the G0/G1 phase of the cell cycle (14). For instance, Diabetic Cardiomyopathy (DCM), defined as the cardiac dysfunction that's characterized with structural, functional and metabolic changes in the myocardium that results in impaired cardiac functions (15). DCM is a distinct entity, first proposed by Lundbaek in 1954, as diabetic heart disease independent of hypertension and coronary artery disease (CAD) that are usually highly prevalent in Diabetes Mellitus (Type-2 Diabetes Mellitus) (16). In this context, chronic low-grade inflammation poses great significance in obesity and T2DM which later showed evidence in contributing to the pathogenesis of DCM (17). Mesenchymal stromal cells have been shown to have anti-diabetic as well as cardioprotective features as mentioned earlier. Studies on the mechanisms of action of mesenchymal cells showed several MSCs functions in DCM (18). One of which is the Anti-Inflammatory feature of MSCs. MSCs influence the infiltration of $\mathrm{T}$ cells in the pancreas along with reducing the cardiac inflammation (18). Moreover, MSCs also have the capacity to decrease cardiac tumor necrosis factor $\alpha$ (TNF- $\alpha$ ) and interleukin-1 expression which both are involved in the initiation and progression of diabetic cardiomyopathy. Hence this anti-inflammatory effect of MSCs protects against myocardial inflammation under Diabetes mellitus (18).

Also, MSCs have an anti-oxidative capacity where MSCs secrete the superoxide dismutase which has the potential to treat diabetic cardiomyopathy (19). This is due to the findings showing that the overexpression of extracellular superoxide dismutase decreases macrophage infiltration and fibrosis thus leading to improved left ventricular function in the diabetic heart (18). This in addition to the anti-fibrotic features of MSCs through reducing cardiac fibrosis via weakening the survival, differentiation, proliferation, and collagen synthesis of cardiac fibroblasts (20). These features are not the only capacities of MSCs on immunomodulatory approaches to Diabetic Cardiomyopathy, 
others include anti-apoptotic features, pro-angiogenic and endothelial-protective features, cardiac progenitor cell-protective features and $\mathrm{Ca}_{2}{ }^{+}$Modulating features (18).

To this end, mesenchymal stem cells "MSC" have been intensively studied as an immunomodulatory approach for the treatment of diabetes and improving the cardiovascular activity. In the context of diabetes research, Bone marrow derived MSCs have been used to form insulin-producing cells and enhance islet engraftment and survival and to treat diabetic ulcers and limb ischemia (21). In experimental models of type 2 diabetes (T2D), the mesenchymal stem cells inoculum improved metabolic control and reduced insulin requirements and of $\mathrm{AlC}$ with no significant opposing results after the intra-arterial injection by selective cannulation of the pancreas vasculature (21).

In a study done by $\mathrm{Si}$ et al., rat models with induced T2D were used to investigate the effects of autologous MSC inoculum. The autologous MSCs were injected shortly "1 or 3 weeks" after the streptozotocin treatment. streptozotocin "STZ" is used to induce a hyperglycaemic state to induce diabetes' (22). The results showed improved metabolic control through enriched insulin secretion, amelioration of insulin insensitivity and increased islet numbers in the pancreas. These results are in consistency with research evidence on the potential therapeutic properties of MSCs to treat diabetes (22). The potential capacity of bone marrow derived MSCs in enhancing the cardiovascular activity in diabetic cardiomyopathy was also investigated and has been shown to lie in its direct differentiation to cardiomyocytes and the ability to secrete potent trophic and paracrine mediators which induces cardiac regeneration and cardio protection (23). Studies on rats with type $1 \mathrm{DM}$, intravenous administration of bonemarrow derived MSCs has shown to improve cardiac function by increasing angiogenesis and attenuating cardiac remodeling. This is attributed to the differentiation of the transplanted MSCs into cardiomyocytes and improved angiogenesis and myogenesis therefore increasing matrix metalloproteinases MMP-2 activity, decreasing that of MMP9 and reducing collagen load in the diabetic myocardium (24).

After this overview of various immunomodulatory approaches related to Cardiovascular Diseases and especially Diabetic Cardiomyopathy, we will look at the immunomodulatory approaches to a Renal Disease: Diabetic Nephropathy (DN). The latter is a syndrome characterized by the presence of pathological quantities of urine albumin excretion, diabetic glomerular lesions, and loss of glomerular filtration rate (GFR), thickening of the glomerular basement membrane $(\mathrm{GBM})$ in diabetic patients (25). Many immunomodulatory approaches are proposed to deal with DN. One of which is also MSCs. In a study done at the Kunming Medical University showed that MSCs ameliorate the renal function and extend survival in diabetic rats, regulate the production of lipoxin 4 (LXA4) and ALX/FPR2 (the receptor of LXA4) in kidney tissue of $\mathrm{DN}$, protect renal function and inhibit fibrosis (26). Other approaches include the novel bifunctional cytokine Interleukin233 (IL-233) that bears IL-2 and IL-33 activities that reverses inflammation and protects against Type 2 Diabetic Nephropathy by promoting T-regulatory cells (Treg) and type 2 immune response. IL-233 also attenuates hyperglycaemia and proteinuria, preserves renal structure and function for long-term and restores glucose clearance and inhibits visceral adiposity (27).

The high prevalence of this syndrome entails the discovery of new treatments to curb the progression of the disease. Because of a better understanding of the disease, there have been many advances in immunomodulatory approaches used for treating diabetes-based cardiorenal diseases. As such, currently there are improvements in the applications of this knowledge to clinical settings, which have led to treatments that are more effective. Since treatments with single agents did not achieve stable metabolic remission as such, future immunomodulatory approaches would focus on dosing, timing, and recognition of the differences between different species. As a result, the next step would be to focus on combined therapy to improve the efficacy of the treatment by promoting additive effects.

In this regard, in what follows we tackle the cardiorenal syndrome; epidemiology \& classification, the relation between the cardiac and renal systems and the pathways involved in cardiorenal syndrome in diabetes namely, RAS, JAK/STAT and oxidative stress.

\section{CARDIORENAL SYNDROME}

The homeostasis in the human body is maintained by the coordinated work of several organs and systems. The most important key players in the homeostasis are the heart and the kidneys. The heart is the pump of the body, which is responsible for circulating blood within the body. On the other hand, the kidneys are responsible for filtering the blood and for the electrolyte homeostasis. These two organs are interlinked; where a dysfunction in one organ affects the other. From this tight relation emerges the cardiorenal syndrome. According to Ronco et al. (28) the cardiorenal syndrome is "the disorders of the heart and kidneys where one organ affects the other." Another more holistic definition was stated by Bock and Gottlieb (29) in their article in which they have mentioned that the cardiorenal syndrome is when "each dysfunctional organ (heart and kidneys) has the ability to initiate and perpetuate disease in the other organ through common hemodynamic, neurohormonal, immunological, and/or biochemical feedback pathways." Although these two definitions have been established around 10 years ago, to this day a clear mechanistic understanding of the cardiorenal syndrome is not yet agreed upon (30).

\section{Epidemiology of Cardiorenal Syndrome}

It is estimated that 25 to $60 \%$ of patients with heart failure have some type of cardiorenal syndrome which is associated with high morbidity and mortality (31). In the United States, the prevalence of chronic heart disease is estimated at $2 \%$ of people over 45 of age. In comparison, in end stage renal disease, $30 \%$ of the patients have chronic heart disease upon the initiation of dialysis (32) and cardiovascular disease is the most common cause of death in those patients (33).

\section{Classifications of Cardiorenal Syndrome}

In 2008, a consensus conference on cardio-renal syndromes was held in Venice Italy under the umbrella of the Acute 
Dialysis Quality Initiative (ADQI) (28). In this conference the classification of the types of the cardiorenal disease were agreed upon. These classifications are considered clinical which lack structural and functional analysis of the disease mechanisms and therapeutic options (34). However, these classifications are useful as a first step to reach a functional classification (35). The classification system is divided into five types of cardiorenal syndrome (28).

\section{Acute Cardio-Renal Syndrome (Type 1)}

This type is characterized by the acute worsening of heart function leading to kidney injury and/or dysfunction. Acute worsening of heart function might include pulmonary oedema, cardiogenic shock, acute heart failure (HF). Approximately 27 to $40 \%$ of patients admitted with acute heart failure develop acute kidney failure (36).

\section{Chronic Cardio-Renal Syndrome (Type 2)}

This type is similar to the first type, except that the heart failure or dysfunction here is chronic which leads to kidney failure. This is the most common type of cardiorenal syndrome. It has been reported that $63 \%$ of patients admitted with congestive heart failure have type 2 cardiorenal syndrome (37).

\section{Acute Cardio-Renal Syndrome (Type 3)}

This type is the opposite of type one, wherein the acute renal impairment causes acute cardiac dysfunction or failure. The epidemiology of this subtype has proven to be a challenge to define due to the wide definition of acute kidney injury and limited reporting on it (28).

\section{Chronic Cardio-Renal Syndrome (Type 4)}

This type is characterized by chronic kidney injury leading to cardiac injury, disease, and/or dysfunction, such as left ventricular failure or diastolic heart failure. There is a strong correlation between the severity of the kidney injury and the unfavorable heart outcomes (38).

\section{Secondary Cardiorenal Syndrome (Type 5)}

This type is characterized by the simultaneous cardiac and renal dysfunction that is caused by a systemic condition that may be chronic or acute. These systemic conditions include sepsis, diabetes mellitus, amyloidosis, and other chronic inflammatory conditions (28).

\section{CARDIORENAL SYNDROME IN DIABETES}

The relation between the heart and the kidneys is a pretty complex one. Many factors, including pathways, molecules, and dysfunctions, come at play to make this tight link. It is hard to study each factor on its own because of their interlinked nature; however, some articles were able to classify these factors into hemodynamic factors and non-hemodynamic factors, as shown in Figure 1 (34). It is important to keep in mind that although this classification is in place, these factors are not independent of each other. It is also evident that these two factors have a strong relation with diabetes. Most of the diabetic complications are due to the macro and microvascular

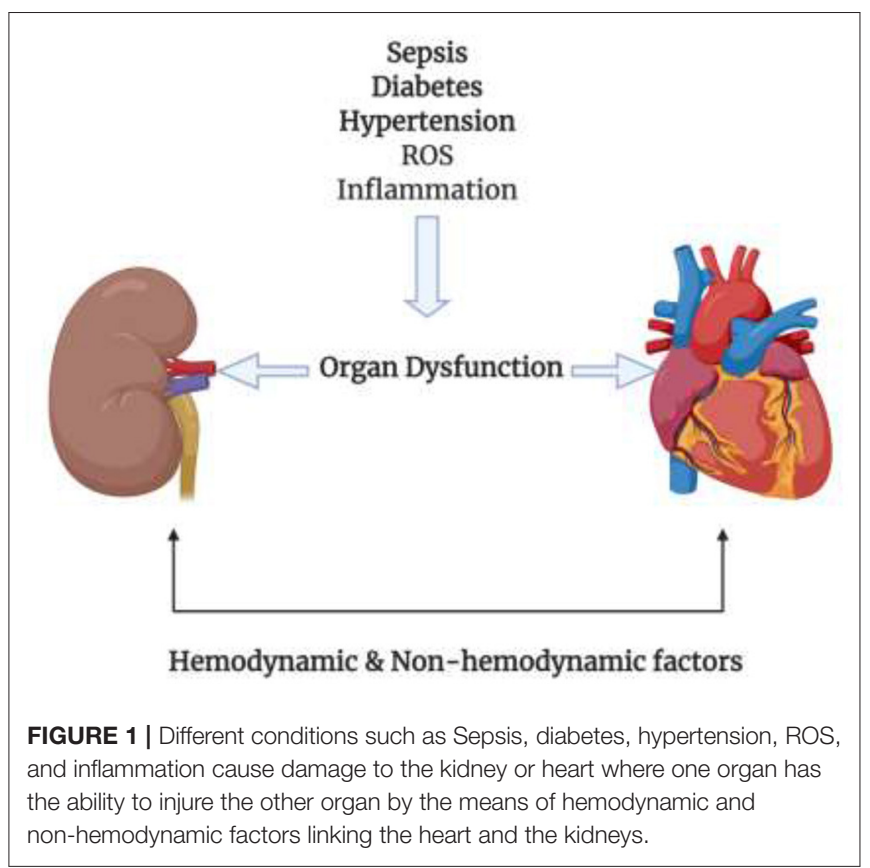

injury, that in turn affect the hemodynamic factors (39). On the other hand, in diabetes the production of mitochondrial ROS plays a huge role in the pathogenesis of diabetes and the development of its complications (40). This lies under the umbrella of non-hemodynamic factors. Moreover, fibrosis is a unifying mechanism linking cardiorenal syndromes. Fibrosis is a result of many metabolic derangements, whether in the heart or in the kidney, which eventually lead to cardiorenal syndromes.

\section{Hemodynamic Factors and Non-hemodynamic Factors Hemodynamic Factors}

Hemodynamics, or in other words dynamics of the blood flow, are tightly controlled by homeostatic mechanisms. Dysfunctions in this well-maintained system have adverse effects on numerous organs and tissues especially the kidneys. The renal blood flow is the primary driver of the glomerular filtration rate (GFR). According to the well-known equation, the GFR equals the renal plasma flow times the filtration fraction. Moreover, many articles that date as back as the mid twentieth century have established that reduced renal blood flow and increased central venous pressure are primary effector mechanisms for renal impairment $(38,41)$. Later studies suggested the renal autoregulation phenomenon, where the reduction in renal blood flow (RBF) was out of proportion to the reduction in cardiac index, while GFR was relatively maintained (42). However, when the renal blood flow drops further, GFR declines as autoregulatory capacity is exhausted (43). In the last few years, the research focus has shifted to the venous congestion as another important factor in the drop of theGFR, independent of the renal blood flow (44). 


\section{Non-hemodynamic Factors}

As stated before, hemodynamic and non-hemodynamic factors are not independent of each other. It is very hard to study the effect of one factor in isolation of the other. Especially that the non-hemodynamic factors, also called cardiorenal connectors, act on the glomerular filtration rate by changes on the hemodynamics. Thus, these cardiorenal connectors are more mediators than direct effectors. The non-hemodynamic factors include a wide range of factors that include the renin angiotensin system (RAS), sympathetic nervous system (SNS) activation, inflammation, endothelial dysfunction.

The RAS is considered a prototypical cardiorenal connector since it is activated bidirectionally by the heart and the kidneys upon failure. Renin is released when renal artery pressure is decreased (45), renal venous pressure is increased (46) and when the delivery of sodium to the distal nephron is decreased which all occur in heart failure and chronic kidney disease. Moreover, angiotensin II has an important effect on renal perfusion and it promotes renal fibrosis, which directly affects GFR, induces hypo-responsiveness to natriuretic peptide and mediates SNS activation (47). The SNS is responsible for altering the ultrafiltration coefficient and is associated with tubular injury and the formation of reactive oxygen species (ROS) (40). Angiotensin II is also responsible for modulating oxidative stress and endothelial dysfunction. Through Nicotinamide Adenine Dinucleotide Phosphate [NADP $(\mathrm{H})]$ activation, angiotensin II promotes the formation of reactive oxygen species, which can cause intrarenal (proximal tubular) damage (33).

\section{Fibrosis as a Unifying Pathophysiology of the Cardiorenal Syndromes}

Fibrosis is a complex cascade of cellular and molecular processes caused by disease related injury. Over a short period of time, fibrosis serves as an adaptive process that helps the organ. However, over an extended period of time, fibrosis will cause parenchymal scarring and ultimately cellular dysfunction and organ failure (48). The main cause of fibrosis in the heart and kidney is inflammation- and oxidative stress-related endothelial dysfunction in aging, hypertension, diabetes mellitus, and obesity (49).

As much as the details described above shows how the heart and kidney are affected, it is of interest to determine the possible pathways that could be implicated in the cardiorenal syndromes.

\section{IMMUNOMODULATORY PATHWAYS INVOLVED IN CARDIORENAL SYNDROME IN DIABETES}

\section{RAS Pathway}

RAS is one of the most important cardiorenal connectors. Improper activation of RAS can lead to both heart and renal failure. In heart failure, RAS alongside the SNS are overactivated (50). Our focus in this section will be Angiotensin II which is the most important effector molecule in the RAS pathway, shown in Figure 2 (51). In response to a drop in blood pressure and/or sodium chloride $(\mathrm{NaCl})$ level,
Renin which is also known as Angiotensinogenase, is secreted by the Juxtaglomerular apparatus in the kidneys (52). It is also secreted in response to SNS activity via the $\beta$-1adrenoceptor activation by norepinephrine secretion which induces inflammation via LPS-induced IL-6 production (5254). Renin acts on Angiotensinogen that is secreted by the liver transforming it to Angiotensin I via hydrolysis; which will then be transformed to Angiotensin II by the action of Angiotensin Converting Enzyme (ACE) that will have multiple effector sites (52). Angiotensin II, upon binding to its AT-1 (Angiotensin II type 1 receptor) and AT-2 receptor (Angiotensin II type 2 receptor) $(53,55)$, induces the production of Interleukin6 (IL-6) and of the TNF- $\alpha$ via the Protein Kinase C (PKC) pathway, followed by the activation of two transcription factors: first of which is the Nuclear Factor Kappa B (NF-кB) through phosphorylation of p65 and then the Activator Protein 1 (AP1) (53). These two transcription factors are important in the pathway for expression of TNF- $\alpha$ and thus for inducing an inflammatory response. This might be an important future perspective in the crosstalk between inflammatory cytokines and RAS in the heart. Another important cytokine induced by Angiotensin II binding to the AT-1 receptor is IL-1 $\beta$. IL$1 \beta$ plays a role in heart failure via systolic dysfunction and ventricular remodeling by upregulating Transforming Growth Factor-Beta (TGF- $\beta$ ) (56). Il-1 $\beta$ impairs systolic function by decreasing the expression of genes important in the regulation of calcium homeostasis (57). It also increases Nitric Oxide Synthase (NOS) expression in cardiac myocytes which leads to an increase in Nitric Oxide (NO) activity, a decrease in energy production and a lower myocardial contractility (57). In addition to that, according to a study performed on diabetic mice, IL-1 $\beta$ leads to cardiac arrhythmia by causing a prolongation of action potential duration, a decrease in potassium current and an increase in Calcium sparks in cardiomyocytes (58). IL$1 \beta$ also affects the synchronized contraction of the heart by decreasing the expression of Connexin $43(\mathrm{Cx} 43)$, a major protein in the cardiac gap junctions (57). Additionally, Angiotensin II induces the production of yet another cytokine, IL-17 via AT-1 receptor binding (59). IL-17, which is produced by T-helper 17 (Th17) cells, triggers the production of other proinflammatory cytokines such as IL- 6 and TNF- $\alpha$, and contributes to the pathogenesis of hypertension and atherosclerosis; as well as, insulin resistance (59).

To add to that, Angiotensin II upregulates the expression of Selectins (P-, E-, and L-selectins), as well as, Vascular Cell Adhesion Molecules-1 (VCAM-1) and Intracellular Adhesion Molecule-1 (ICAM-1), via TNF- $\alpha(55,60)$. These markers are elevated in Chronic Kidney Disease and Chronic Heart Failure (60). Angiotensin II also increases the kidney expression of Endothelin 1 (ET-1) (61), which stimulates vasoconstriction, inflammation, and fibrosis (cardiac remodeling) (62). Thus, this expression is increased in hypertension, heart failure, and kidney disease. Another role of Angiotensin II in oxidative stress, apoptosis, and inflammation is via Toll-Like Receptor 4 (TLR4) expression via binding to AT-1 receptor (55). The TLR4 signaling inflammatory cascade mediates renal dysfunction via phosphorylation of Extracellular Receptor Kinase (ERK) and 


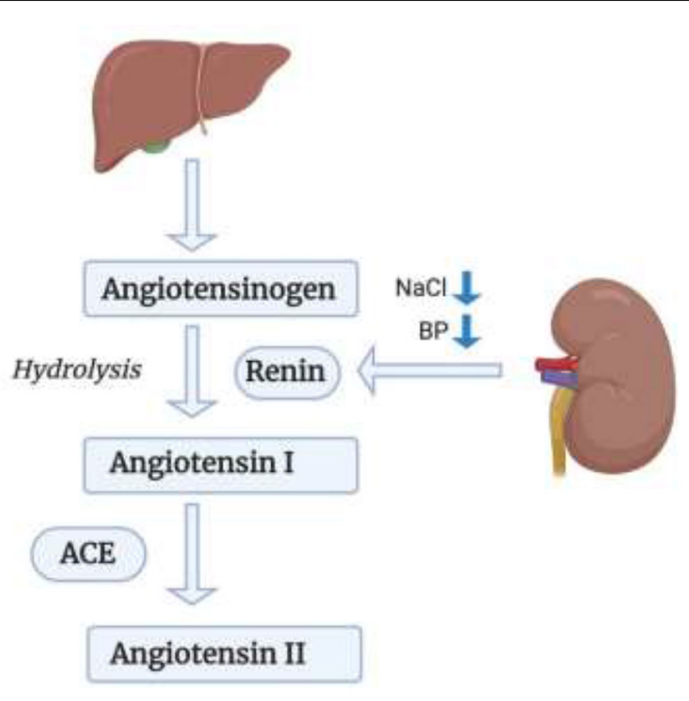

FIGURE 2 | RAS pathway in leading kidney failure. Renin acts on Angiotensinogen secreted by the liver, transforming it to Angiotensin I, which will then be transformed into Angiotensin II via ACE.

Mitogen Activated Protein Kinase (MAPK) (63). The MAPK signaling is also activated by Angiotensin II. Furthermore, Angiotensin II plays a role in oxidative stress via Cyclooxygenase 2 (COX-2) activation to generate vasoactive prostaglandins and ROS which will play a role in endothelial dysfunction (55). It also generates ROS upon binding to its AT-1 receptor via NADPH oxidase (NOX) (55). A summary of some signaling pathways of Angiotensin II is shown in Figure 3.

\section{JAK/STAT Pathway}

IL-6, which is also activated by Angiotensin II, is secreted as a result of ischemia, and binds to plasma membrane receptors which leads to a signal transduction pathway activating JAK and STAT proteins (64). This signaling pathway has an important role in diabetic nephropathy via an Angiotensin IIdependent mechanism, and is negatively regulated by Suppressor of Cytokine Signaling 3 (SOCS3) by either inhibiting the JAK tyrosine kinase activity or by competing with STATs on cytokine receptors (65). This means SOCS3 negatively regulates IL-6. Another protein mediated by STAT3 is Ephrin-B2, which is increased in diabetes (66). Ephrin-B2 stimulates cardiac fibrosis by the activation and interaction of STAT3 and TGF- $\beta /$ SMAD 3 signaling pathways (67). IL- 6 and TNF- $\alpha$ are also regulated by the interaction between Lipopolysaccharide (LPS) and Cluster of Differentiation 14 (CD14) via activation of Nuclear Factor Kappa $\mathrm{B}(\mathrm{NF}-\kappa \mathrm{B})$ signaling (68).

\section{ROS INDUCED INFLAMMATORY AND CARDIORENAL SYNDROMES IN DIABETES}

Oxidative Stress is described as an imbalance between oxidants (like ROS) and antioxidants (like NO), which results in an accumulation of the oxidants (69). So, it is when the production of oxidants (ROS) is greater than the body's antioxidative metabolic ability. ROS play a major role in hypertension, cardiovascular disease, and renal damage, which emphasizes their contribution to cardiorenal syndrome (as shown in Figure 4) (55). ROS are small molecules derived from oxygen; they are generated in several cellular processes. One major way is Angiotensin II-induced activation of NADPH oxidase, as we mentioned before.

Nicotinamide Adenine Dinucleotide Phosphate (NADPH) oxidase, also known as NOX, plays a major role in ROS formation via utilizing $\mathrm{NADPH}$ as an electron donor to reduce oxygen and produce superoxide anions $\left(\mathrm{O}_{2}^{-}\right)$and Hydrogen Peroxide $\left(\mathrm{H}_{2} \mathrm{O}_{2}\right)$ (70). The high levels of oxygen radicals lead to mitochondrial dysfunction by inactivating mitochondrial enzymes and cause DNA damage at the cellular level (71). At the organ level, our focus will be on the cardiorenal axis and markers implicated. The imbalance between ROS and $\mathrm{NO}$ is one of the most reliable markers of oxidative stress. The decrease in NO can be due mainly to two things: Reaction of NO with oxygen radicals and high concentrations of Asymmetric Dimethyl Arginine (ADMA) (33). Superoxide anion $\left(\mathrm{O}_{2}^{-}\right)$reacts with $\mathrm{NO}$ to form Peroxynitrite $\left(\mathrm{ONOO}^{-}\right)$, the accumulation of which results in vasoconstriction, inflammation, and impaired vascular and renal functions (70). This decrease in NO inhibits P450 enzymes and leads to the production of vasoconstriction molecules (72) via enhancing Cyclooxygenase activity, which promotes the production of Thromboxane A2 (TxA2) (vasoconstrictor) (72). Also, Peroxynitrite increases Thromboxane synthase activity and thus TxA2, so it increases the vasoconstrictor; and inhibits Prostacyclin synthase and thus decreases Prostacyclin-2 production (vasodilator); which leads to an imbalance between the vasoconstrictors and vasodilators which contributes to the pathogenesis of both the heart and the kidney (72). Another factor contributing to the decrease of $\mathrm{NO}$ is the high concentration of circulating ADMA, which is an endogenous inhibitor of Nitric Oxide Synthase (NOS) and is highly observed in renal failure (73).

On the other hand, in heart failure, mitochondrial dysfunction plays a role via Angiotensin II by upregulating NOX2; we also have a Mitochondrial-ROS independent pathway by Angiotensin II that results in the upregulation of NOX4 (71). In addition to that, ROS activates Matrix Metalloproteinase (MMP) in cardiac fibroblasts, which leads to structural changes in the myocardium (74). This leads to cardiac remodeling, decrease in contractility, dysfunctional Calcium handling and eventually heart failure (74). Moreover, Hyperuricemia, which is the accumulation of uric acid mainly due to malfunctioning Xanthine Oxidase (XO), also leads to oxidative stress via a dysfunction in the release of ROS and NO. This is associated with kidney disease, heart disease and diabetes (75). ROS also has proinflammatory effect by releasing cytokines; in addition to profibrotic effect by inducing EpithelialMesenchymal Transition (EMT) via the Mitogen-Activated Protein kinase (MAPK) activation or SMAD signaling and this will lead to renal fibrosis, in addition to cellular hypertrophy via Extracellular Receptor Kinase (ERK1/ERK2) pathways (70). These most important proinflammatory cytokines are TL-6 and TNF-alpha, and the main transcription factor responsible for 


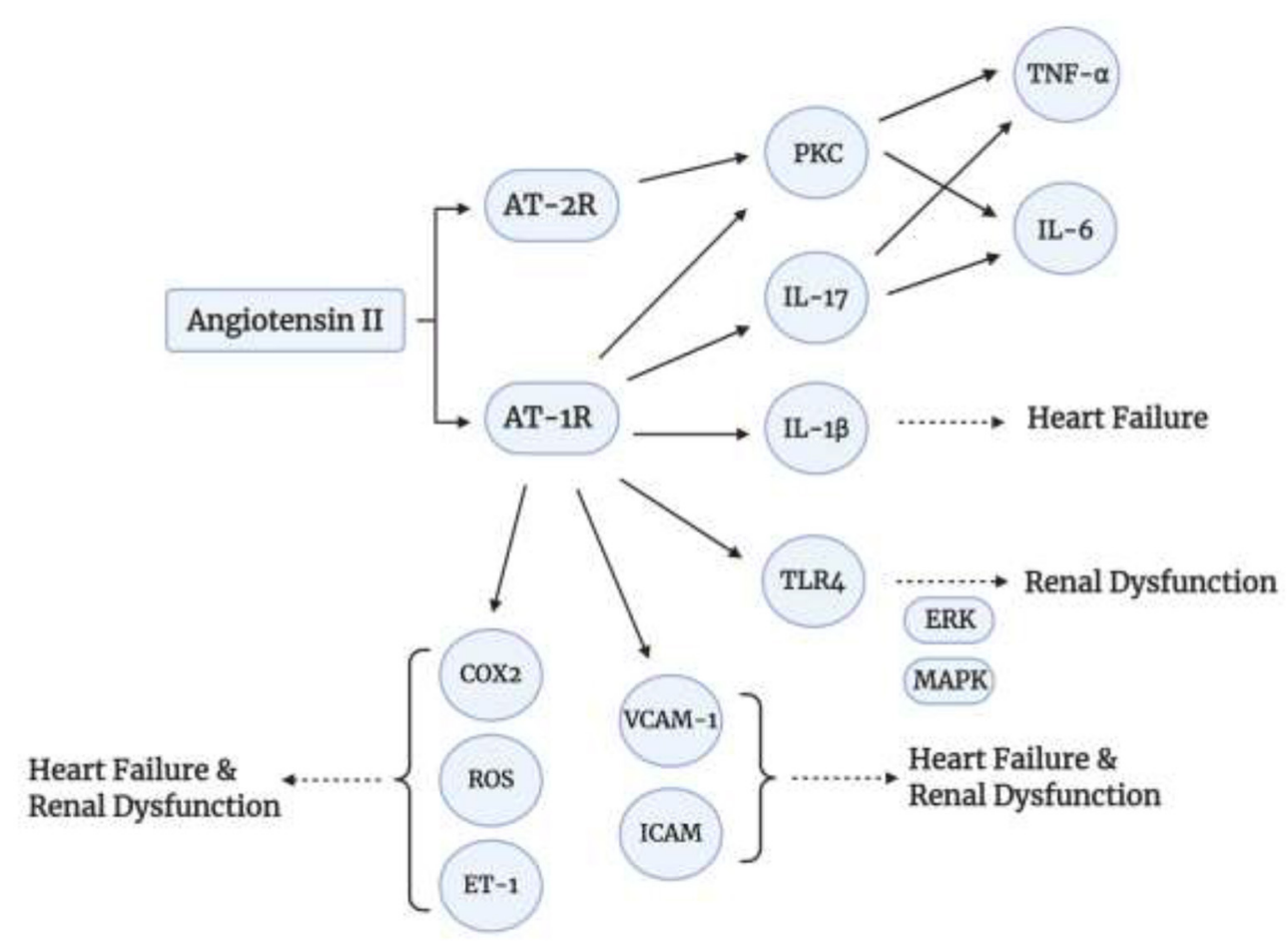

FIGURE 3 | Angiotensin II signaling pathways. Angiotensin II induces inflammatory cytokine production via binding to AT-1 and AT-2.

initiating the proinflammatory response is NF-kB, the expression of which is increased in diabetic experimental models (76). So, the downstream effects of NF-kB are proinflammatory via MCP-1, TNF-alpha, and IL-6 regulation. Furthermore, at the level of the mitochondria, excess-glucose leads to increased glucose-derived pyruvate oxidation which increases the number of electron donors in the electron transport chain resulting in over production of superoxide (76).

\section{ANTIOXIDANTS AND ANTI-INFLAMMATORY APPROACHES TO TREAT DIABETES-INDUCED CARDIORENAL SYNDROME}

As discussed previously and evidenced in the literature, oxidative stress has a huge role in injury and pathogenesis of cardiorenal syndrome (77). Therefore, it logically entails that antioxidants have a possible protective ability that would prevent cardiorenal syndrome. The role of oxidative stress and antioxidants appears clearly in diabetes mellitus related cardiorenal syndrome (type 5) (78). Therefore, we will focus in this part on evidence that has explained and proven the protective role of antioxidants in diabetes mellitus and type 5 cardiorenal syndrome. Since the 1960s, the role of antioxidants in improving health and wellbeing was discovered by biologists (79). In the next decade, Cameron and Pauling (80) were able to find that ascorbic acid (vitamin C) is a potential human cancer protective agent.
Since then, antioxidants have become a hot topic in medical research and scientists are looking deeper into the mechanisms, molecular targets, and molecular interactions of antioxidants (81). The antioxidant defense mechanisms are divided into 2 categories: enzymatic and non-enzymatic strategies. Enzymatic antioxidants include superoxide dismutase, catalase, glutathione peroxidase, and glutathione reductase. While non-enzymatic antioxidants include the vitamins $\mathrm{A}, \mathrm{C}$, and $\mathrm{E}$, glutathione, $\alpha$ lipoic acid, mixed carotenoids, coenzyme Q10 (CoQ10), several bioflavonoids, antioxidant minerals (copper, zinc, manganese and selenium), and cofactors like folic acid, uric acid, albumin (82).

In his experiment, Kunisaki et al. (83) was able to show that when he administered vitamin $\mathrm{E}$ to diabetic rats, the retinal blood flow and PKC activity in the vascular tissue were normalized. Also, another two short-term experimental studies proved that high doses of vitamin $\mathrm{C}$ and lipoic acid can improve some aspects of endothelial dysfunction in diabetes $(84,85)$. Furthermore, it has been recently reported that vitamin $\mathrm{E}$ has the ability to reduce the oxidative stress that builds up in the macrophages in diabetic mice (86). Finally, other studies were able to find prophylactic effects of vitamin $\mathrm{E}$ on heart failure patients that have type 1 diabetes. They were able to show that the "supplementation of streptozotocin-induced (STZ)-diabetic rats with $2000 \mathrm{IU}$ of vitamin E/kg of feed beginning immediately after induction of DM and continuing for 8 weeks provided significant protection against cardiac dysfunction induced by T1DM" (87). 


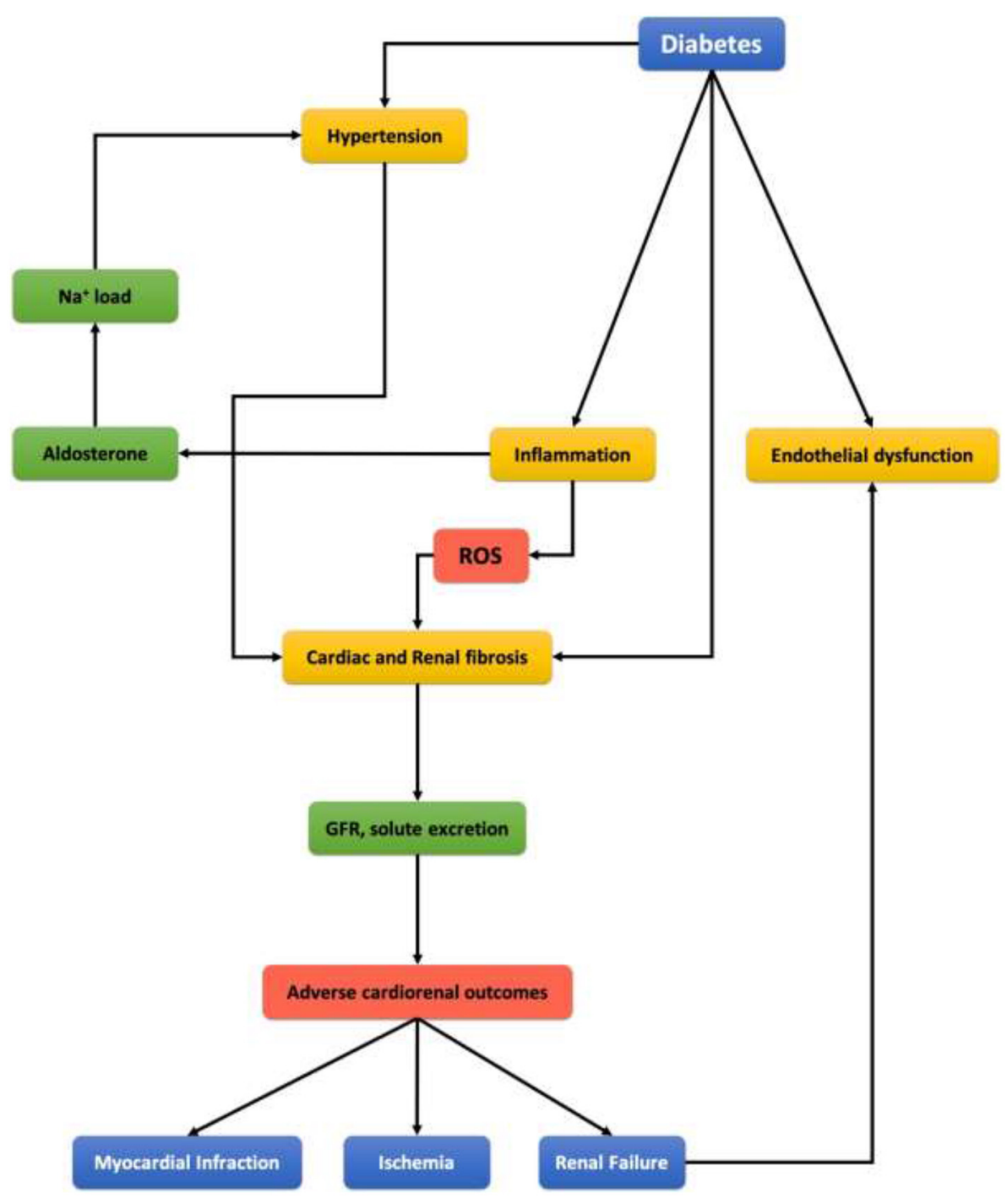

FIGURE 4 | Representing scheme of clinical link between diabetes mellitus, cardiovascular disease, and chronic kidney disease.

As previously revisited mesenchymal stem cells "MSCs"; these cells have the capacity to self-renew rendering them an important immunomodulatory approach to autoimmune diseases (88). MSCs produce soluble factors that can increase the production of anti-inflammatory cytokine interleukin (IL) -10 and decreased production of interferon-gamma (IFN- $\gamma$ ) and IL -12 through altering the secretion of dendritic cells (DCs) $(89,90)$ MSCs engage the inhibitory molecule programmed death 1 (PD-1) to its ligands PD-L1 and PD-L2 thus, suppressing T-cell proliferation $(90,91)$. This control over T-cells including $\mathrm{CD} 4^{+} \mathrm{CD} 25^{+} \mathrm{FoxP}^{+}(90)$, has influence on the susceptibility to diabetes induction (92) Moreover MSCs, through the production of soluble factors can also inhibit the proliferation and secretion of B cells (90). This release of trophic and immunomodulatory factors by MSCs seems to hold the therapeutic capacity of these cells (90).
Another therapeutic approach for the cardiorenal syndrome is via the Chinese herbal medication Qiliqiangxin (QLQX). QLQX is composed of 11 different herbs that are alismatis rhizome, carthami flos, cinnamomi ramulus, ginseng radix et rhizome, astragali radix, citri reticulatae pericarpium, salvia miltiorrhiza radix et rhizome, aconiti lateralis radix preparata, semen descurainiae lepidii, periploca cortex, and polygonati odorati rhizome (93). It has been shown that QLQX can be used for regulating the immune response and improving circulation via the Astragali Radix component and acts by reducing the production of TNF- $\alpha$ (93). In addition to that, QLQX has a similar effect as Olmesartan by inhibiting the AT-1 receptor; and thus, inhibits the Ang II-induced cardiac fibroblasts' transdifferentiation via reducing IL-6 transcription and regulating nuclear activity of Nuclear Factor of Activated T-cells (NFAT3) (94). By that, QLQX attenuates cardiac 
inflammatory reactions and protects myocardial structure and function in HF (94). On the other hand, QLQX can also protect against renal injury in cardiorenal syndrome (CRS) by regulating the oxidative stress and inflammation signaling (95). QLQX significantly reduced inflammatory cytokines and AT receptors in the kidney reducing the inflammatory response; in addition to, reducing the ROS content and thereby regulating the oxidative stress response (95).

Additionally, a study has shown that QLQX improves autophagy via TRPV-1 dependent mechanism in the diabetic heart (9). It also showed that QLQX treatment improves cardiac function and myocardial phenotype in the diabetic mouse model (96). To add to that, QLQX improves endothelial aortic function in diabetic rats via RAS and NO pathways; by inhibiting the expression of ACE and AT-1, and by regulating the NO balance (97).

\section{CONCLUSION}

CRS defines the different clinical conditions in which heart dysfunction and kidney dysfunction overlap, it describes the negative effects of an impaired renal function on the heart and circulation. In this review, we have thoroughly explained CRS beginning from epidemiological data and classifications aiming

\section{REFERENCES}

1. Nijnik A, Immunomodulatory approaches for prevention and treatment of infectious diseases. Curr Opin Microbiol. (2013) 16:590-5. doi: 10.1016/j.mib.2013.06.011

2. Bascones-Martinez A, Mattila R, Gomez-Font R, Meurman JH. Immunomodulatory drugs: oral and systemic adverse effects. Med Oral Patol Oral Cir Bucal. (2014) 19:e24-31. doi: 10.4317/medoral.19087

3. Clarke JO, Mullin GE. A review of complementary and alternative approaches to immunomodulation. Nutr Clin Pract. (2008) 23:49-62. doi: 10.1177/011542650802300149

4. Khalil DN, Smith EL, Brentjens RJ, Wolchok JD. The future of cancer treatment: immunomodulation, CARs and combination immunotherapy. Nat Rev Clin Oncol. (2016) 13:394. doi: 10.1038/nrclinonc.2016.65

5. Panahi M, Papanikolaou A, Torabi A, Zhang JG, Khan H, Vazir A, et al. Immunomodulatory interventions in myocardial infarction and heart failure: a systematic review of clinical trials and meta-analysis of IL-1 inhibition. Cardiovasc Res. (2018) 114:1445-61. doi: 10.1093/cvr/cvy145

6. Pino CJ, Westover AJ, Johnston KA, Buffington DA, Humes HD. Regenerative medicine and immunomodulatory therapy: insights from the kidney, heart, brain, and lung. Kidney Int Rep. (2018) 3:77183. doi: 10.1016/j.ekir.2017.12.012

7. Rosano GM, Vitale C, Seferovic P. Heart failure in patients with diabetes mellitus. Card Fail Rev. (2017) 3:52-5. doi: 10.15420/cfr.2016:20:2

8. Fadini GP, Avogaro A, Degli Esposti L, Russo P, Saragoni S, Buda S, et al. Risk of hospitalization for heart failure in patients with type 2 diabetes newly treated with DPP-4 inhibitors or other oral glucose-lowering medications: a retrospective registry study on 127,555 patients from the Nationwide OsMed Health-DB Database. Eur Heart J. (2015) 36:245462. doi: 10.1093/eurheartj/ehv301

9. de Candia P, Prattichizzo F, Garavelli S, De Rosa V, Galgani M, Di Rella F, et al. Type 2 diabetes: how much of an autoimmune disease? Front Endocrinol. (2019) 10:451. doi: 10.3389/fendo.2019.00451

10. Zhou Y, Yamamoto Y, Xiao Z, Ochiya T. The immunomodulatory functions of mesenchymal stromal/stem cells mediated via paracrine activity. J Clin Med. (2019) 8:1025. doi: $10.3390 / \mathrm{jcm} 8071025$ to clarify the historical lack of a clear definition due to the complexity of this disease. Immunomodulation encompasses all therapeutic interventions aimed at modifying the immune response as such offering innovative drug targets and methods for immunomodulatory interventions. A better understanding of CRS holds the promise of regenerative medicine, which points toward repairing damaged tissues, stimulating the healing mechanism of organs and implanting laboratory grown tissues when the body is unable to heal itself.

\section{AUTHOR CONTRIBUTIONS}

$\mathrm{AE}$ and $\mathrm{FH}$ conceived and designed the review article and approved the final version to be submitted. LA, MN, and NS performed the literature review and wrote the first draft of the review. HG discussed, assembled the data, and revised the manuscript for intellectual content. NA worked on the figures of this review. All authors contributed to the article and approved the submitted version.

\section{ACKNOWLEDGMENTS}

We would like to thank all the members of the Eid Laboratory and the AUB diabetes. Figures created with BioRender.com.

11. Ben-Ami E, Berrih-Aknin S, Miller, A. Mesenchymal stem cells as an immunomodulatory therapeutic strategy for autoimmune diseases. Autoimmun Rev. (2011) 10:410-5. doi: 10.1016/j.autrev.2011.01.005

12. Di Nicola M, Carlo-Stella C, Magni M, Milanesi M, Longoni PD, Matteucci P, et al. Human bone marrow stromal cells suppress T-lymphocyte proliferation induced by cellular or nonspecific mitogenic stimuli. Blood. (2002) 99:383843. doi: 10.1182/blood.V99.10.3838

13. Aggarwal S, Pittenger MF. Human mesenchymal stem cells modulate allogeneic immune cell responses. Blood. (2005) 105:1815-22. doi: 10.1182/blood-2004-04-1559

14. Glennie S, Soeiro I, Dyson PJ, Lam EW, Dazzi F. Bone marrow mesenchymal stem cells induce division arrest anergy of activated T cells. Blood. (2005) 105:2821-7. doi: 10.1182/blood-2004-09-3696

15. Athithan L, Gulsin GS, McCann GP, Levelt E. Diabetic cardiomyopathy: Pathophysiology, theories and evidence to date. World J Diabetes. (2019) 10:490-510. doi: 10.4239/wjd.v10.i10.490

16. Lundbaek K. Diabetic angiopathy: a specific vascular disease. Lancet. (1954) 266:377-9. doi: 10.1016/S0140-6736(54)90924-1

17. Borghetti G, von Lewinski D, Eaton DM, Sourij H, Houser SR, Wallner M. Diabetic cardiomyopathy: current and future therapies. Beyond glycemic control. Front Physiol. (2018) 9:1514. doi: 10.3389/fphys.2018.01514

18. Linthout SV, Spillmann F, Schultheiss HP, Tschöpe C. Effects of mesenchymal stromal cells on diabetic cardiomyopathy. Curr Pharm Des. (2011) 17:33417. doi: 10.2174/138161211797904163

19. Kemp K, Gray E, Mallam E, Scolding N, Wilkins A. Inflammatory cytokine induced regulation of superoxide dismutase 3 expression by human mesenchymal stem cells. Stem Cell Rev Rep. (2010) 6:54859. doi: 10.1007/s12015-010-9178-6

20. Mias C, Lairez O, Trouche E, Roncalli J, Calise D, Seguelas MH, et al. Mesenchymal stem cells promote matrix metalloproteinase secretion by cardiac fibroblasts and reduce cardiac ventricular fibrosis after myocardial infarction. Stem Cells. (2009) 27:2734-43. doi: 10.1002/stem.169

21. Pileggi A. Mesenchymal stem cells for the treatment of diabetes. Diabetes. (2012) 61:1355-6. doi: 10.2337/db12-0355

22. Si Y, Zhao Y, Hao H, Liu J, Guo Y, Mu Y, et al. Infusion of mesenchymal stem cells ameliorates hyperglycemia in type 2 diabetic rats: identification 
of a novel role in improving insulin sensitivity. Diabetes. (2012) 61:161625. doi: $10.2337 / \mathrm{db} 11-1141$

23. Davey GC, Patil SB, O'Loughlin A, O'Brien T. Mesenchymal stem cell-based treatment for microvascular and secondary complications of diabetes mellitus. Front Endocrinol. (2014) 5:86. doi: 10.3389/fendo.2014.00086

24. Zhang N, Li J, Luo R, Jiang J, Wang JA. Bone marrow mesenchymal stem cells induce angiogenesis and attenuate the remodeling of diabetic cardiomyopathy. Exp Clin Endocrinol Diabetes. (2008) 116:104-11. doi: 10.1055/s-2007-985154

25. Lim A. Diabetic nephropathy - complications and treatment. Int J Nephrol Renovasc Dis. (2014) 7:361-81. doi: 10.2147/IJNRD.S40172

26. Bai Y, Wang J, He Z, Yang M, Li L, Jiang H. Mesenchymal stem cells reverse diabetic nephropathy disease via lipoxin $\mathrm{A} 4$ by targeting transforming growth factor beta (TGF-beta)/smad pathway and pro-inflammatory cytokines. Med Sci Monit. (2019) 25:3069-76. doi: 10.12659/MSM.914860

27. Sabapathy V, Stremska ME, Mohammad S, Corey RL, Sharma PR, Sharma R. Novel immunomodulatory cytokine regulates inflammation, diabetes, and obesity to protect from diabetic nephropathy. Front Pharmacol. (2019) 10:572. doi: 10.3389/fphar.2019.00572

28. Ronco C, McCullough P, Anker SD, Anand I, Aspromonte N, Bagshaw $\mathrm{SM}$, et al. Cardio-renal syndromes: report from the consensus conference of the acute dialysis quality initiative. Eur Heart J. (2010) 31:70311. doi: 10.1093/eurheartj/ehp507

29. Bock JS, Gottlieb SS. Cardiorenal syndrome: new perspectives. Circulation. (2010) 121:2592-600. doi: 10.1161/CIRCULATIONAHA.109.886473

30. Damman K, Testani JM. The kidney in heart failure: an update. Eur Heart J. (2015) 36:1437-44. doi: 10.1093/eurhearti/ehv010

31. Ronco C, Di Lullo L. Cardiorenal syndrome in Western Countries: epidemiology, diagnosis and management approaches. Kidney Dis. (2017) 2:151-63. doi: $10.1159 / 000448749$

32. Harnett JD, Foley RN, Kent GM, Barre PE, Murray D, Parfrey PS. Congestive heart failure in dialysis patients: prevalence, incidence, prognosis and risk factors. Kidney Int. (1995) 47:884-90. doi: 10.1038/ki.1995.132

33. Bongartz LG, Cramer MJ, Doevendans PA, Joles JA, Braam B. The severe cardiorenal syndrome: 'guyton revisited'. Eur Heart J. (2005) 26:117. doi: 10.1093/eurheartj/ehi020

34. Braam B, Joles JA, Danishwar AH, Gaillard CA. Cardiorenal syndromecurrent understanding and future perspectives. Nat Rev Nephrol. (2014) 10:48-55. doi: 10.1038/nrneph.2013.250

35. Hatamizadeh P, Fonarow GC, Budoff MJ, Darabian S, Kovesdy CP, Kalantar-Zadeh K. Cardiorenal syndrome: pathophysiology and potential targets for clinical management. Nat Rev Nephrol. (2013) 9:99-111. doi: 10.1038/nrneph.2012.279

36. Gottlieb SS, Abraham W, Butler J, Forman DE, Loh E, Massie BM, et al. The prognostic importance of different definitions of worsening renal function in congestive heart failure. J Card Fail. (2002) 8:13641. doi: $10.1054 /$ jcaf.2002.125289

37. Heywood JT, Fonarow GC, Costanzo MR, Mathur VS, Wigneswaran JR, Wynne J, et al. High prevalence of renal dysfunction and its impact on outcome in 118,465 patients hospitalized with acute decompensated heart failure: a report from the ADHERE database. J Card Fail. (2007) 13:42230. doi: 10.1016/j.cardfail.2007.03.011

38. Hill NR, Fatoba ST, Oke JL, Hirst JA, O'Callaghan CA, Lasserson DS, et al. Chronic kidney disease and mortality risk: a systematic review. J Am Soc Nephrol. (2006) 17:2034-47. doi: 10.1681/ASN.2005101085

39. Mota RI, Morgan SE, Bahnson EM. Diabetic vasculopathy: macro and microvascular injury. Curr Pathobiol Rep. (2020) 8:1-14. doi: 10.1007/s40139-020-00205-x

40. Nishikawa T, Araki E. Impact of mitochondrial ROS production in the pathogenesis of diabetes mellitus and its complications. Antioxid Redox Signal. (2007) 9:343-53. doi: 10.1089/ars.2006.1458

41. Maxwell MH, Breed ES, Schwartz IL. Renal venous pressure in chronic congestive heart failure. J Clin Invest. (1950) 29:342-8. doi: 10.1172/JCI102263

42. Ljungman S, Laragh JH, Cody RJ. Role of the kidney in congestive heart failure. Relationship of cardiac index to kidney function. Drugs. (1990) 39 (Suppl 4):10-21; discussion 22-4. doi: 10.2165/00003495-199000394-00004

43. Smilde TD, Damman K, van der Harst P, Navis G, Westenbrink BD, Voors $\mathrm{AA}$, et al. Differential associations between renal function and "modifiable" risk factors in patients with chronic heart failure. Clin Res Cardiol. (2009) 98:121-9. doi: 10.1007/s00392-008-0732-Z

44. Mullens W, Abrahams Z, Francis GS, Sokos G, Taylor DO, Starling RC, et al. Importance of venous congestion for worsening of renal function in advanced decompensated heart failure. J Am Coll Cardiol. (2009) 53:58996. doi: 10.1016/j.jacc.2008.05.068

45. Kirchheim H, Ehmke H, Persson P. Sympathetic modulation of renal hemodynamics, renin release and sodium excretion. Klin Wochenschr. (1989) 67:858-64. doi: 10.1007/BF01717340

46. Kishimoto T, Maekawa M, Abe Y, Yamamoto K. Intrarenal distribution of blood flow and renin release during renal venous pressure elevation. Kidney Int. (1973) 4:259-66. doi: 10.1038/ki.1973.112

47. Kon V, Yared A, Ichikawa I. Role of renal sympathetic nerves in mediating hypoperfusion of renal cortical microcirculation in experimental congestive heart failure and acute extracellular fluid volume depletion. J Clin Invest. (1985) 76:1913-20. doi: 10.1172/JCI112187

48. Rockey DC, Bell PD, Hill JA. Fibrosis-a common pathway to organ injury and failure. N Engl J Med. (2015) 373:96-5. doi: 10.1056/NEJMc1504848

49. Travers JG, Kamal FA, Robbins J, Yutzey KE, Blaxall BC. Cardiac fibrosis: the fibroblast awakens. Circ Res. (2016) 118:102140. doi: 10.1161/CIRCRESAHA.115.306565

50. Zucker IH, Xiao L, Haack KK. The central renin-angiotensin system and sympathetic nerve activity in chronic heart failure. Clin Sci. (2014) 126:695706. doi: $10.1042 / C S 20130294$

51. Crowley, SD, Rudemiller NP. Immunologic effects of the renin-angiotensin system. J Am Soc Nephrol. (2017) 28:1350-61. doi: 10.1681/ASN.2016101066

52. Patel S, Rauf A, Khan H, Abu-Izneid T. Renin-angiotensin-aldosterone (RAAS): The ubiquitous system for homeostasis and pathologies. Biomed Pharmacother. (2017) 94:317-25. doi: 10.1016/j.biopha.2017.07.091

53. Kalra D, Sivasubramanian N, Mann DL. Angiotensin II induces tumor necrosis factor biosynthesis in the adult mammalian heart through a protein kinase C-dependent pathway. Circulation. (2002) 105:2198205. doi: 10.1161/01.CIR.0000015603.84788.47

54. Liao J, Keiser JA, Scales WE, Kunkel SL, Kluger MJ. Role of epinephrine in TNF and IL-6 production from isolated perfused rat liver. Am J Physiol. (1995) 268 (4 Pt. 2):R896-901. doi: 10.1152/ajpregu.1995.268.4.R896

55. Benigni A, Cassis P, Remuzzi G. angiotensin II revisited: new roles in inflammation, immunology and aging. EMBO Mol Med. (2010) 2:24757. doi: 10.1002/emmm.201000080

56. Liu Q, Wang $\mathrm{T}, \mathrm{Yu} \mathrm{H}$, Liu B, Jia R. Interaction between interleukin-1 beta and angiotensin II receptor 1 in hypothalamic paraventricular nucleus contributes to progression of heart failure. J Interferon Cytokine Res. (2014) 34:870-5. doi: 10.1089/jir.2013.0159

57. Szekely Y, Arbel Y. A review of interleukin-1 in heart disease: where do we stand today? Cardiol Ther. (2018) 7:25-44. doi: 10.1007/s40119-018-0104-3

58. Monnerat G, Alarcón ML, Vasconcellos LR, Hochman-Mendez C, Brasil G, Bassani RA, et al. Macrophage-dependent IL-1 $\beta$ production induces cardiac arrhythmias in diabetic mice. Nat Commun. (2016) 7:13344. doi: $10.1038 /$ ncomms13344

59. Ohshima K, Mogi M, Jing F, Iwanami J, Tsukuda K, Min LJ, et al. Roles of interleukin 17 in angiotensin II type 1 receptor-mediated insulin resistance. Hypertension. (2012) 59:493-9. doi: 10.1161/HYPERTENSIONAHA.111.183178

60. Colombo PC, Ganda A, Lin J, Onat D, Harxhi A, Iyasere JE, et al. Inflammatory activation: cardiac, renal, and cardio-renal interactions in patients with the cardiorenal syndrome. Heart Fail Rev. (2012) 17:17790. doi: 10.1007/s10741-011-9261-3

61. Komers R, Plotkin H. Dual inhibition of renin-angiotensinaldosterone system and endothelin-1 in treatment of chronic kidney disease. Am J Physiol Regul Integr Comp Physiol. (2016) 310:R877-84. doi: 10.1152/ajpregu.00425.2015

62. Kohan DE. Endothelin, hypertension and chronic kidney disease: new insights. Curr Opin Nephrol Hypertens. (2010) 19:134-9. doi: 10.1097/MNH.0b013e328335f91f

63. Nair AR, Elks CM, Vila J, Del Piero F, Paulsen DB, Francis J. A blueberryenriched diet improves renal function and reduces oxidative stress in metabolic syndrome animals: potential mechanism of TLR4-MAPK signaling pathway. PLoS ONE. (2014) 9:e111976. doi: 10.1371/journal.pone.0111976 
64. Boengler K, Hilfiker-Kleiner D, Drexler H, Heusch G, Schulz R. The myocardial JAK/STAT pathway: from protection to failure. Pharmacol Ther. (2008) 120:172-85. doi: 10.1016/j.pharmthera.2008.08.002

65. Matsui F, Meldrum KK. The role of the Janus kinase family/signal transducer and activator of transcription signaling pathway in fibrotic renal disease. J Surg Res. (2012) 178:339-45. doi: 10.1016/j.jss.2012.06.050

66. Coucha M, Barrett AC, Elgebaly M, Ergul A, Abdelsaid M. Inhibition of ephrin-B2 in brain pericytes decreases cerebral pathological neovascularization in diabetic rats. PLoS ONE. (2019) 14:e0210523. doi: 10.1371/journal.pone.0210523

67. Su SA, Yang D, Wu Y, Xie Y, Zhu W, Cai Z, et al. EphrinB2 regulates cardiac fibrosis through modulating the interaction of Stat3 and TGF- $\beta /$ Smad3 signaling. Circ Res. (2017) 121:61727. doi: 10.1161/CIRCRESAHA.117.311045

68. Popko K, Gorska E, Stelmaszczyk-Emmel A, Plywaczewski R, Stoklosa A, Gorecka D, et al. Proinflammatory cytokines Il- 6 and TNF- $\alpha$ and the development of inflammation in obese subjects. Eur J Med Res. (2010) 15 (Suppl. 2):120-2. doi: 10.1186/2047-783X-15-S2-120

69. Giam B, Kaye DM, Rajapakse NE. Role of renal oxidative stress in the pathogenesis of the cardiorenal syndrome. Heart Lung Circ. (2016) 25:87480. doi: 10.1016/j.hlc.2016.02.022

70. Rubattu S, Mennuni S, Testa M, Mennuni M, Pierelli G, Pagliaro B, et al. Pathogenesis of chronic cardiorenal syndrome: is there a role for oxidative stress? Int J Mol Sci. (2013) 14:23011-32. doi: 10.3390/ijms141123011

71. Newsholme P, Haber EP, Hirabara SM, Rebelato EL, Procopio J, Morgan D, et al. Diabetes associated cell stress and dysfunction: role of mitochondrial and non-mitochondrial ROS production and activity. J Physiol. (2007) 583(Pt. 1):9-24. doi: 10.1113/jphysiol.2007.135871

72. Liaudet L, Soriano FG, Szabó C. Biology of nitric oxide signaling. Crit Care Med. (2000) 28(4 Suppl):N37-52. doi: 10.1097/00003246-200004001-00005

73. Vallance P, Leone A, Calver A, Collier J, Moncada S. Accumulation of an endogenous inhibitor of nitric oxide synthesis in chronic renal failure. Lancet. (1992) 339:572-5. doi: 10.1016/0140-6736(92)90865-Z

74. Spinale FG, Coker ML, Thomas CV, Walker JD, Mukherjee R, Hebbar L. Time-dependent changes in matrix metalloproteinase activity and expression during the progression of congestive heart failure: relation to ventricular and myocyte function. Circ Res. (1998) 82:482-95. doi: 10.1161/01.RES.8 2.4.482

75. Riegersperger M, Covic A, Goldsmith D. Allopurinol, uric acid, and oxidative stress in cardiorenal disease. Int Urol Nephrol. (2011) 43:4419. doi: 10.1007/s11255-011-9929-6

76. Jha JC, Banal C, Chow BS, Cooper ME, Jandeleit-Dahm K. Diabetes and kidney disease: role of oxidative stress. Antioxid Redox Signal. (2016) 25:657684. doi: 10.1089/ars.2016.6664

77. Virzì GM, Clementi A, de Cal M, Brocca A, Day S, Pastori S, et al. Oxidative stress: dual pathway induction in cardiorenal syndrome type 1 pathogenesis. Oxid Med Cell Longev. (2015) 2015:391790. doi: 10.1155/2015/391790

78. Matough FA, Budin SB, Hamid ZA, Alwahaibi N, Mohamed J. The role of oxidative stress and antioxidants in diabetic complications. Sultan Qaboos Univ Med J. (2012) 12:5-18. doi: 10.12816/0003082

79. Rowe LA, Degtyareva N, Doetsch PW. DNA Damage-Induced Reactive Oxygen Species: A Genotoxic Stress Response. Atlanta, GA: Emory University (2009).

80. Cameron E, Pauling L. Supplemental ascorbate in the supportive treatment of cancer: Prolongation of survival times in terminal human cancer. Proc Natl Acad Sci USA. (1976) 73:3685-9. doi: 10.1073/pnas.73.10.3685

81. American Institute for Cancer Research. Food, Nutrition, Physical Activity, and the Prevention of Cancer: a Global Perspective. Washington, DC: American Institute for Cancer Research (1997).

82. Maritim AC, Sanders RA, Watkins JB III. Diabetes, oxidative stress, and antioxidants: a review. J Biochem Mol Toxicol. (2003) 17:2438. doi: 10.1002/jbt.10058

83. Kunisaki M, Bursell SE, Clermont AC, Ishii H, Ballas LM, Jirousek MR, et al. Vitamin E prevents diabetes-induced abnormal retinal blood flow via the diacylglycerol-protein kinase C pathway. Am J Physiol. (1995) 269 (2 Pt. 1):E239-46. doi: 10.1152/ajpendo.1995.269.2.E239

84. Mohora M, Vîrgolici B, Coman A, Muscurel C, Găman L, Gruia V, et al. Diabetic foot patients with and without retinopathy and plasma oxidative stress. Rom J Intern Med. (2007) 45:51-7.

85. Obrosova IG, Fathallah L, Greene DA. Early changes in lipid peroxidation and antioxidative defense in diabetic rat retina: effect of DL-alpha-lipoic acid. Eur J Pharmacol. (2000) 398:139-46. doi: 10.1016/S0014-2999(00)00286-7

86. Hayek T, Kaplan M, Kerry R, Aviram M. Macrophage NADPH oxidase activation, impaired cholesterol fluxes, and increased cholesterol biosynthesis in diabetic mice: a stimulatory role for D-glucose. Atherosclerosis. (2007) 195:277-86. doi: 10.1016/j.atherosclerosis.2006.12.026

87. Hamblin M, Smith HM, Hill MF. Dietary supplementation with vitamin E ameliorates cardiac failure in type I diabetic cardiomyopathy by suppressing myocardial generation of 8-iso-prostaglandin F2alpha and oxidized glutathione. J Card Fail. (2007) 13:884-92. doi: 10.1016/j.cardfail.2007.07.002

88. Volarevic V, Arsenijevic N, Lukic ML, Stojkovic M. Concise review: mesenchymal stem cell treatment of the complications of diabetes mellitus. Stem Cells. (2011) 29:5-10. doi: 10.1002/stem.556

89. Abdi R, Fiorina P, Adra CN, Atkinson M, Sayegh MH. Immunomodulation by mesenchymal stem cells: a potential therapeutic strategy for type 1 diabetes. Diabetes. (2008) 57:1759-67. doi: 10.2337/db08-0180

90. Volarevic V, Al-Qahtani A, Arsenijevic N, Pajovic S, Lukic ML. Interleukin1 receptor antagonist (IL-1Ra) and IL-1Ra producing mesenchymal stem cells as modulators of diabetogenesis. Autoimmunity. (2010) 43:25563. doi: 10.3109/08916930903305641

91. Nauta AJ, Fibbe WE. Immunomodulatory properties of mesenchymal stromal cells. Blood. (2007) 110:3499-506. doi: 10.1182/blood-2007-02-069716

92. Zdravkovic N, Shahin A, Arsenijevic N, Lukic ML, Mensah-Brown EP. Regulatory $\mathrm{T}$ cells and ST2 signaling control diabetes induction with multiple low doses of streptozotocin. Mol Immunol. (2009) 47:2836. doi: 10.1016/j.molimm.2008.12.023

93. Xiao H, Song Y, Li Y, Liao YH, Chen J. Qiliqiangxin regulates the balance between tumor necrosis factor-alpha and interleukin-10 and improves cardiac function in rats with myocardial infarction. Cell Immunol. (2009) 260:515. doi: 10.1016/j.cellimm.2009.09.001

94. Zhou J, Jiang K, Ding X, Fu M, Wang S, Zhu L, et al. Qiliqiangxin inhibits angiotensin II-induced transdifferentiation of rat cardiac fibroblasts through suppressing interleukin-6. J Cell Mol Med. (2015) 19:111421. doi: $10.1111 / \mathrm{jcmm} .12512$

95. Duan X, Yan F, Hu H, Liu H, Wu Q, Sun S, et al. Qiliqiangxin protects against renal injury in rat with cardiorenal syndrome type i through regulating the inflammatory and oxidative stress signaling. Biol Pharm Bull. (2018) 41:1178-85. doi: 10.1248/bpb.b17-00930

96. Tong J, Lai Y, Yao YA, Wang XJ, Shi YS, Hou HJ, et al. Qiliqiangxin rescues mouse cardiac function by regulating AGTR1/TRPV1-mediated autophagy in STZ-induced diabetes mellitus. Cell Physiol Biochem. (2018) 47:136576. doi: 10.1159/000490822

97. Chen F, Wu JL, Fu GS, Mou Y, Hu SJ. Chronic treatment with qiliqiangxin ameliorates aortic endothelial cell dysfunction in diabetic rats. J Cardiovasc Pharmacol Ther. (2015) 20:230-40. doi: 10.1177/1074248414537705

Conflict of Interest: The authors declare that the research was conducted in the absence of any commercial or financial relationships that could be construed as a potential conflict of interest.

Copyright (c) 2021 Ammar, Nahlawi, Shayya, Ghadieh, Azar, Harb and Eid. This is an open-access article distributed under the terms of the Creative Commons Attribution License (CC BY). The use, distribution or reproduction in other forums is permitted, provided the original author(s) and the copyright owner(s) are credited and that the original publication in this journal is cited, in accordance with accepted academic practice. No use, distribution or reproduction is permitted which does not comply with these terms. 